\title{
Evaluation of antimicrobial prescription pattern in Neonatal Intensive care unit of tertiary care teaching hospital
}

\author{
Borade $S^{1}$, Ghodki $S^{2}$, Bhore $A^{3}$, Tiwari $S^{4}$, Jahagirdar $S^{5}$, Bansod $K^{6}$ \\ ${ }^{1}$ Dr. Sanjio Borade, Senior Resident, Department of Pharmacology, ${ }^{2}$ Dr Sarang Ghodki, Senior Resident, Department of \\ Pharmacology, ${ }^{3}$ Dr. Ashok Bhore, Professor and Head, Dept. of Pharmacology, ${ }^{4}$ Dr. Satish Tiwari, Professor and Head, \\ Dept. of Paediatrics, ${ }^{5}$ Dr. Shreepad Jahagirdar, Associated Professor, Department of Paediatrics, ${ }^{6}$ Dr. Kishor \\ Bansod, Associated Professor, Department of Pharmacology. All are affiliated to Dr. Panjabrao Deshmukh Memorial \\ Medical College, Amravati.
}

Address for correspondence: Dr Sanjino Borade, Email: sanjioborade@gmail.com

\begin{abstract}
Introduction: Neonates admitted in Neonatal Intensive Care Units (NICU) have high morbidity and mortality with very subtle and subjective clinical signs. Hence/so Anti Microbial Agents (AMAs), being/become mainstay drugs, are often used empirically and irrationally. Method: This is cross- sectional study over the period of six months from October 2012 to March 2013. Clinical, hematologic, laboratory, microbiologic and therapeutic data were collected, analyzed and evaluated from the case papers of NICU. Rational use in our study means appropriate dose, duration, frequency and route of administration appropriate to clinical conditions. Result: Of 118 neonates, 66 (56\%) were treated rationally. Approximately $60 \%$ times appropriate dose and frequency of drugs were given. Cefotaxime was most commonly prescribed AMA for neonates $(73.73 \%)$. In our study low birth weight neonates have received more antibiotics in comparison with term babies. Conclusion: AMA prescription policy be formulated and displayed in NICU to promote rational prescription.
\end{abstract}

Key words: Neonatal intensive care unit, Neonates, Empiric, Rational AMA Prescription Policy.

\section{Introduction}

Neonatal Intensive Care Unit (NICU) is a setup where good number of neonates with expectant high morbidity and mortality are admitted. They are treated frequently with antimicrobial agents (AMAs) for varied indications such as Septicemia, Urinary Tract Infection, Respiratory Tract infection, Necrotizing Enterocolitis and Meningitis. All these increase the cost of treatment. [1,2]

AMAs are the mainstay drugs in NICU. Hence appropriate use of AMA in infection and treatment is very crucial. While prescribing, not only the knowledge of Pharmacology (Pharmacokinetics - Absorption, Distribution, Metabolism, Excretion and drug interactions) but also that of Gestational maturity and weight of neonate, patho-physiology of disease, correct diagnosis, microbiological pattern, adverse drug reaction and approach in selecting cost effective drug matters $[3,4]$. It is a known fact that AMAs are used indiscriminately, excessively or inadequately.

Manuscript received: $14^{\text {th }}$ Aug 2014

Reviewed: $07^{\text {th }}$ Sept 2014

Author Corrected: $24^{\text {th }}$ Sept 2014

Accepted for Publication: $7^{\text {th }}$ Oct 2014
Apart from these therapeutically significant aspects, it results in emergence of microbial resistance. [3,5-9]. This additionally increases the cost of treatment along with morbidity and mortality resulting from drugs.

So appropriate use of AMAs is of great significance in clinical practice $[4,10]$. This may help medical care to be more effective, rational and cost effective.

Hence, we planned to conduct a study on AMAs prescribed to the patients admitted in the NICU of the Department of Pediatrics to study the rational i.e. appropriate use of AMAs on the basis of dose and frequency $\&$ to derive the recommendations based on the observations.

\section{Material and methods}

Present cross- sectional study was conducted at Neonatal intensive care Unit (NICU) of Dr. Panajbrao Deshmukh Medical College and hospital Amravati. Data was collected from case papers of NICU available in Medical Record Section of the hospital. Total 227 neonates 
admitted in the NICU during $1^{\text {st }}$ October 2012 to $31^{\text {st }}$ March 2013. Among these, who received AMAs were included in the study and those who did not receive were excluded.

The information from the case records (clinical findings and laboratory data) was collected, evaluated and analyzed for rationality. [11] (Co-relation with clinical, hematologic, microbiological and radiological data, along with accuracy of prescribed doses, durations and frequency of administration of AMAs.)

We studied the AMAs administration mainly on the basis of dose and frequency of administration. There are no well defined guidelines for the adequacy of duration of AMA therapy. Hence we did not take into account the duration AMA administration[2].

Instead of number of neonates, number of times AMAs administration were considered. The AMA prescription data was compared and analyzed with reference to the guidelines of The Harriet Lane Handbook, 19 ${ }^{\text {th }}$ Ed.[12] which is the standard reference book in neonatology followed in the institute.

There is no antibiotic policy existing in NICU of the institute.

Ethical clearance: An ethical approval was obtained from Institutional Ethical Committee.

\section{Indications for AMA use:}

1) Empiric therapy: based on clinical diagnosis only [3].

2) Empiric therapy with supportive laboratory data (Hematologic and CRP) [3]

3) Targeted therapy: based on positive blood culture sensitivity report [3].

In our study Rationality means appropriate use of AMAs on the basis of dose and frequency. $[12,13,14]$

Data Analysis: Descriptive statistics like proportions, mean, standard deviation were calculated. The analysis was done with the help of statistical software open Epi version 2.3 .

\section{Results}

Of 118 neonates, 63 were male and 55 female. Mean age was 5.4 (days), mean gestational age was 36.3 (wks.), mean birth wt. was 2.1(kg.), mean wt. on admission $2 \mathrm{~kg}$. Average duration of stay in hospital was 9.8 (Days) and Number of AMAs per neonate was 2.059 .

In our study, most common co-morbid condition in neonates admitted in NICU was Septicemia 47 (39.83\%), followed by Respiratory distress 31 (26.27\%). Neonates admitted for Pre-term care and other co-morbid conditions were $11(9.32 \%)$ each. Hyperbilirubinemia was seen in $08(06.78 \%)$ neonates.

While only 5 (04.24\%) neonates were admitted with birth asphyxia and Meconium Stained Amniotic fluid as co-morbid conditions in each.

Table 1: AMAs prescribed to neonates admitted in NICU

\begin{tabular}{|c|l|c|c|}
\hline SN & AMA & No. of pts. $(\mathbf{n}=\mathbf{1 1 8})$ & \% \\
\hline 1 & Cefotaxime & 87 & 73.73 \\
\hline 2 & Amikacin & 44 & 37.29 \\
\hline 3 & Piperacillin & 30 & 25.42 \\
\hline 4 & Meropenem & 18 & 15.25 \\
\hline 5 & Amoxicillin + clavulanic acid & 17 & 14.41 \\
\hline 6 & Vancomycin & 11 & 9.32 \\
\hline
\end{tabular}

Total 19 AMAs were prescribed. Cefotaxime was most commonly prescribed in 87 (35.8\%) of neonates, followed by Amikacin in 44 (18.11\%), Piperacillin in 30 (12.3) and Meropenem in 18 (7.41\%). Other less prescribed drugs include Ciprofloxacin, linezolid, ceftazidime, ceftriaxone \& fluconazole. 
Fig 1: Number of AMAs prescribed to neonates

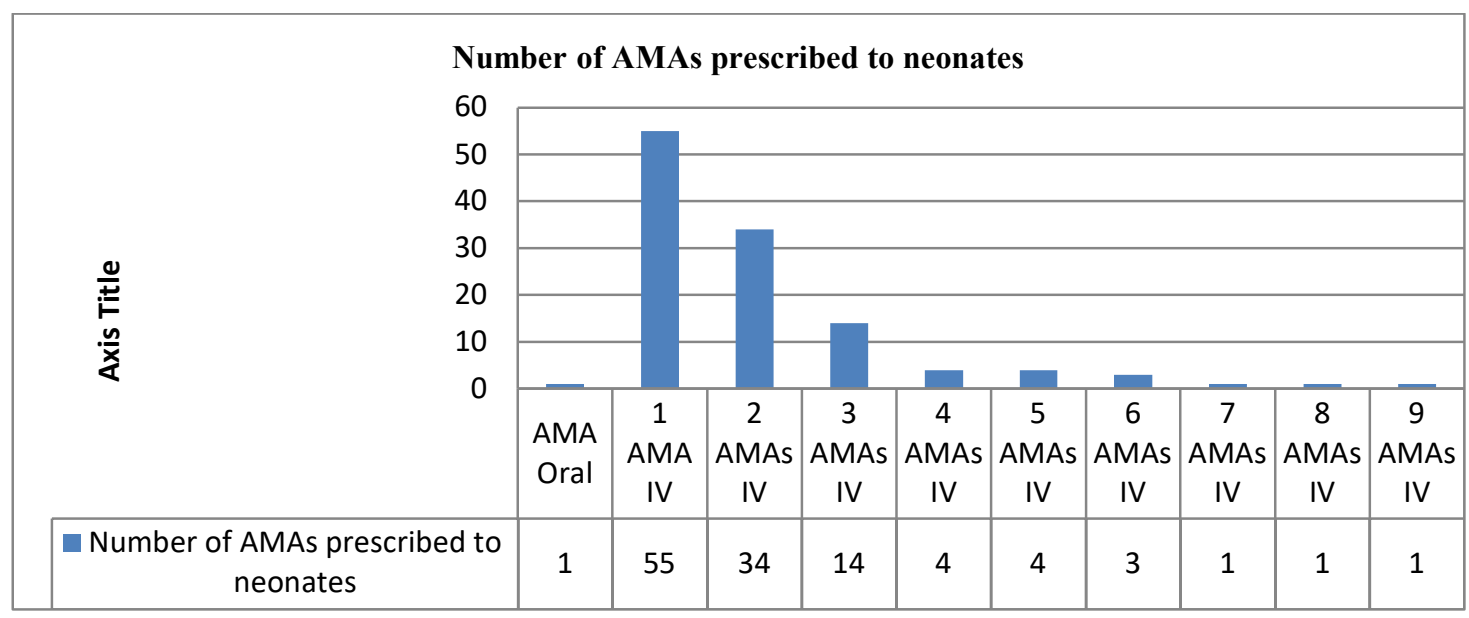

55 (46.61\%) neonates received single IV AMA, followed by 2 AMAs in 34 (28.81\%), 3 AMAs in 14(11.86\%). Only 1 $(0.85 \%)$ neonate received single oral AMA.

Table 2: Use of AMAs in neonates according to appropriate dose and frequency

\begin{tabular}{|c|l|c|c|c|c|c|}
\hline SN & \multirow{2}{*}{ AMAs } & \multicolumn{3}{|c|}{ No. of times Doses given } & \multicolumn{2}{c|}{ Freq. of Dose administration } \\
\cline { 3 - 7 } & & Total & Appropriate & Inappropriate & Appropriate & Inappropriate \\
\hline 1 & Cefotaxim & 92 & $71(77.17)$ & $21(22.83)$ & $71(77.17)$ & $21(22.83)$ \\
\hline 2 & Amikacin & 44 & $27(61.36)$ & $17(38.64)$ & $27(61.36)$ & $17(38.64)$ \\
\hline 3 & $\begin{array}{l}\text { Piperacillin }+ \\
\text { Tazobactam }\end{array}$ & 30 & $6(20)$ & $24(80)$ & $16(53.33)$ & $14(46.67)$ \\
\hline 4 & Meropenem & 19 & $14(73.68)$ & $5(26.32)$ & $13(68.42)$ & $6(31.58)$ \\
\hline 5 & $\begin{array}{l}\text { Amoxicillin + clavulanic } \\
\text { acid }\end{array}$ & 17 & $16(94.12)$ & $1(5.88)$ & $17(100)$ & $0(00)$ \\
\hline 6 & Vancomycin & 11 & $8(72.72)$ & $3(27.27)$ & $6(54.55)$ & $5(45.46)$ \\
\hline
\end{tabular}

This table shows, of 92 times cefotaxim administered, based on doses criteria, 71 times it was appropriate and 21 times it was inappropriate and same was true on the basis of freq. Piperaciline + tazobactum were administered 30 times. On dose basis it was administered appropriately for 6 times and inappropriately for 24 times, whereas on frequency basis it was administered appropriately for 16 times and inappropriately for 14 times.

Meropenem was administered 19 times. On dose basis it was administered appropriately for 14 times and inappropriately for 05 times, whereas on freq. basis it was administered appropriately for 13 times and inappropriately for 06 times. Ampiciline is administered appropriately on the basis of both dose and frequency.

Table 3: Pattern of Administration of AMAs According to dose and frequency

\begin{tabular}{|c|c|c|c|c|}
\hline SN. & Per Dose & Frequency & No. of Doses & \% \\
\hline 1 & Same & Same & 145 & 59.67 \\
\hline 2 & $\uparrow /$ Same & $\uparrow$ & $17+20$ & 15.23 \\
\hline 3 & $\uparrow$ & Same & 22 & 09.47 \\
\hline 4 & $\downarrow$ & Same & 08 & 09.05 \\
\hline 5 & $\downarrow$ & $\uparrow$ & 03 & 03.29 \\
\hline 6 & $\uparrow$ & $\downarrow$ & 03 & 1.23 \\
\hline 7 & Same & $\downarrow$ & 02 & 1.23 \\
\hline 8 & Same (Total dose) & $\uparrow / \downarrow$ & $\mathbf{2 4 3}$ & 0.83 \\
\hline
\end{tabular}




\section{Research Article}

In this study we closely scrutinized the accuracy of AMA administration on the basis of dose and frequency. AMAs were administered $145(59.67 \%$ ) times with appropriate dose and frequency. In remaining $40 \%$ times either dose or frequency was inappropriate.

\section{Table 4: Hematologic data}

\begin{tabular}{|c|c|c|c|c|c|c|c|c|c|c|}
\hline \multicolumn{2}{|c|}{$\begin{array}{l}\text { Haematological } \\
\text { Data }(n=118)\end{array}$} & \multicolumn{3}{|c|}{$\begin{array}{l}\text { C- Reactive Protein } \\
\text { (CRP) }(\mathrm{n}=118)\end{array}$} & \multicolumn{6}{|c|}{$\begin{array}{l}\text { Haematologic data (Total leukocyte count and absolute neutrophil } \\
\text { count ) \& CRP }(n=118)\end{array}$} \\
\hline $\begin{array}{l}\text { Supp } \\
\text { ortive }\end{array}$ & $\begin{array}{l}\text { Non } \\
\text { Suppor } \\
\text { tive }\end{array}$ & $+\mathrm{ve}$ & -ve & $\begin{array}{l}\text { Not } \\
\text { Don } \\
\mathrm{e}\end{array}$ & $\begin{array}{c}\text { Both } \\
\text { - ve }\end{array}$ & $\begin{array}{l}\text { Both } \\
+ \text { ve }\end{array}$ & $\begin{array}{c}\text { Haem. } \\
\text { - ve \& } \\
\text { CRP + } \\
\text { ve }\end{array}$ & $\begin{array}{l}\text { Haem. } \\
\text { + ve \& } \\
\text { CRP - } \\
\text { ve }\end{array}$ & $\begin{array}{c}\text { Only Haemat } \\
+ \text { ve (CRP } \\
\text { not done) }\end{array}$ & $\begin{array}{c}\text { Only } \\
\text { Haemat. - ve } \\
\text { (CRP not } \\
\text { done) }\end{array}$ \\
\hline 32 & 86 & 45 & 49 & 24 & 37 & 16 & 29 & 12 & 04 & 20 \\
\hline $\begin{array}{c}27.12 \\
\%\end{array}$ & $\begin{array}{c}72.88 \\
\%\end{array}$ & $\begin{array}{c}38.14 \\
\%\end{array}$ & $\begin{array}{c}41.53 \\
\%\end{array}$ & $\begin{array}{l}20.3 \\
4 \%\end{array}$ & $\begin{array}{l}31.36 \\
\%\end{array}$ & $\begin{array}{c}13.56 \\
\%\end{array}$ & $24.58 \%$ & $10.17 \%$ & $03.39 \%$ & $16.95 \%$ \\
\hline
\end{tabular}

We classified the patients treated with AMAs according to clinical diagnosis in the group of empiric therapy.

Of 118 neonates, 61 (51.69\%) showed laboratory (haematological and CRP) data positive. We grouped them as empiric with supportive laboratory data.

Hematologic and CRP were negative in 37 (31.36\%), and both were positive in 16 (13.56\%) neonates. AMAs were prescribed in 37 patients on clinical ground even if there were no evidences to support diagnosis of sepsis. There were 29 $(24.58 \%)$ cases in which Hematologic data was negative and CRP was positive. On the other hand, in 12 (10.17\%) neonates hematologic data was positive but CRP was negative.

Table 5: Distribution of patients advised blood culture sensitivity admitted in NICU

\begin{tabular}{|l|l|l|l|}
\hline Blood culture Sensitivity & & No. of Patients & $\mathbf{\%}$ \\
\hline C/S done & + ve & 13 & 11.02 \\
\cline { 2 - 4 } & - ve & 2 & 01.69 \\
\hline C/S Not Done & & 103 & 87.29 \\
\hline & Total & 118 & 100 \\
\hline
\end{tabular}

From the total 118 neonates, blood culture was done in $15(12.71 \%), 13$ of them showed positive culture sensitivity report and were treated accordingly and appropriately. We classified them in targeted therapy.

Fig 2: Number of neonates received AMAs

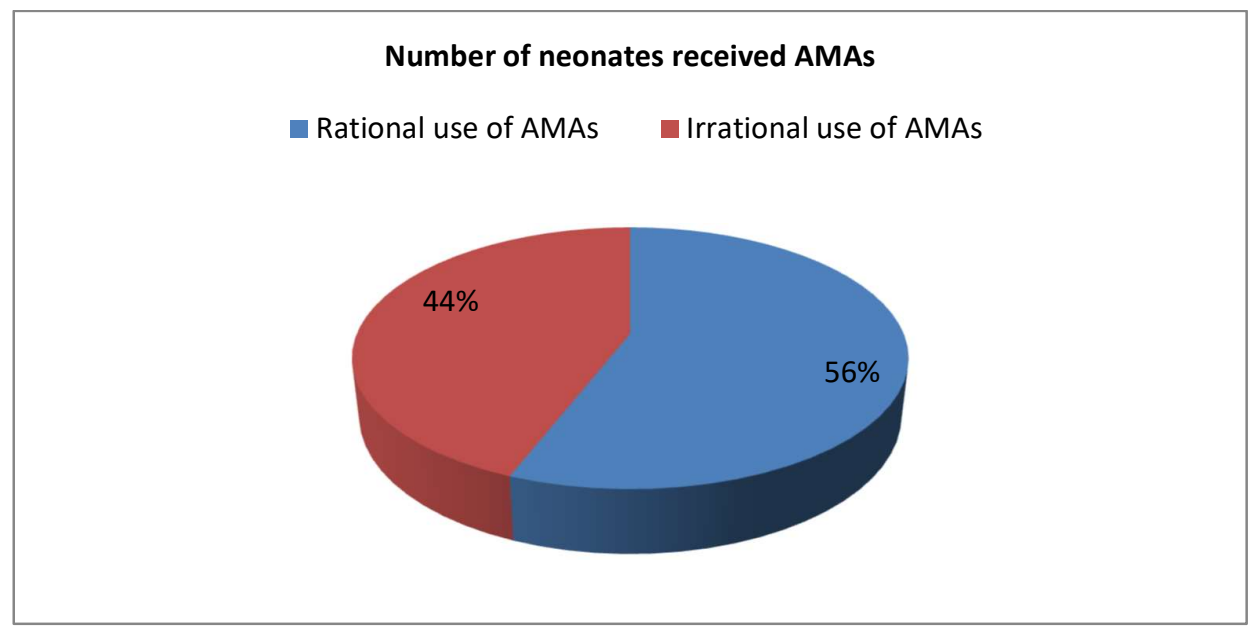

Of 118 neonates, 66 (56\%) were treated rationally. 


\section{Discussion}

As per WHO definition of rational drug therapy "Ppatients receive medications appropriate to their clinical needs, in doses that meet their own individual requirements, for an adequate period of time, and at the lowest cost to them and their community." (WHO, 1985) [13].In this definition, the significance of laboratory investigations in appropriate diagnosis of clinical condition is not clearly defined. [13]

Empiric antimicrobial use is defined as antimicrobial therapy begun when a physician treats the suspected infection only clinically without microbiologic and laboratory data [3]. This may lead to either excessive or inadequate use of AMAs. On the other hand, if clinical diagnosis is well supported by laboratory data then it may result in rational appropriate administration of AMAs appropriate for clinical condition [3].

Clinical diagnosis alone may not be adequate. It is better, if supported by hematologic, laboratory and microbiologic investigations. All these make the AMA administration rational. If AMAs are given in subtherapeutic frequency and doses, disease may deteriorate and prolong. AMAs given in supra-therapeutic dose, may result in toxicity. Also AMAs, given either for short or prolonged duration, may lead to development of microbial resistance.

In hospital based practice, in the present scenario of judicial activism, one must keep in mind issues related to i) medical negligence and consumer's protection act [14] and ii) evidence based medicine.

While scrutinizing AMAs on the basis of rationality, we mainly focused on individual dose, total dose and frequency of administration. Of 118 neonates, only 66 $(55.93 \%)$ were treated rationally. We did not take into account the duration of AMA administration, since no clear- cut guidelines for duration of administration for infection are defined except for meningitis and septicemia [2].

Mean birth wt. was $2.1 \mathrm{~kg}$. In similar study it was $1.69 \mathrm{~kg}$ at the time of admission [2]. Duration of stay in hospital was $9.8 \pm 9.17$ (Days). In similar study it was 29.8 (Days) [2]. Average number of AMAs prescribed per patient during study period was 2.059. In similar study it was 3.4 [2].

In preterm neonates, pharmacokinetic efficiencies like drug absorption, distribution, metabolism and excretion are not fully matured. This demands tailor- made adjustment in dose and frequency. In our study, every dose was calculated for every neonate on the basis of dose and frequency as per the guidelines of The Harriet Lane Handbook, $19^{\text {th }}$ Ed [12].

In our study, Cefotaxime, Amikacin and Piperacillin were given to 87,44 and 30 neonates. In similar study, these AMAs were given to 5, 66 and 62 respectively [2].

In our study, the number of AMAs administered per patient ranged from one to nine antibiotics which is similar to other study [2].

In similar study on AMA prescription in NICU, CRP is regularly recommended investigation in diagnosis as well as in deciding AMA [6]. In our study, it was not done in $24(20.34 \%)$ neonates. In our setup, majority of neonates receive AMAs even before admission. Due to this, the chances of getting blood culture sensitivity positive become very less. Hence next best option is to rely upon hematologic data and CRP for deciding administration of AMAs.

In targeted therapy, AMAs are administered according to blood culture and sensitivity report which is gold standard practice. It is routine for all neonates in other studies [3,4]. It was meagerly done in our study (15 cases). In similar studies, among AMAs macrolides are used for the treatment of atypical pneumonias [2] which are not used at all in our study.

It was observed, of 243 times the doses administered, 168 times AMAs were administered in appropriate doses and frequency [13].

\section{Conclusion}

AMAs are very important drugs . They should be administered in neonatal units with great precautions, taking into account gestational age, wt. on admission, severity of infection- judged by clinical assessment, hematologic data, microbiologic data. In calculating doses, pharmacokinetics of different gestational ages must be taken into consideration which greatly affects the dose and frequency of administration. All these flaws can be minimized by forming an antibiotic policy or protocol which should be displayed in working place for staff, residents and nurses.

Most of the studies and literature define rationality on the basis of dose, frequency and duration. No well defined guidelines are available to define appropriateness of clinical condition. And due to this, there remains confusion as to how much importance to be given to 
laboratory data in considering rationality. In our study and review of available literature we observed that, in considering rationality - in addition to dose, duration and frequency of administration of AMAs, laboratory investigation findings should be given a thought $[16,17]$.

\section{Funding: Nil \\ Conflict of interest: Nil \\ Permission from IRB: Yes}

\section{References}

1. Marlies A. van Houten, Marian Laseur, Jan L.L. Kimpen. Shift in antibiotic prescribing patterns, in relation to antibiotic expenditure in paediatrics, European Journal of Paediatrics 1998;157(6):479-481.

2. Schellack N, Gous AGS. Antibiotic prescribing patterns in a neonatal intensive care unit. South Afr J Epidemiol Infect 2011;26(4)(Part II):267-270.

3. Josiah Olusegun Alamu. Evaluation of antimicrobial use in a paediatric Intensive care unit, University of Iowa, Iowa Research Online 2009, http://ir.uiowa.edu assessed on 01-08-2014.

4. Shah MN, Desai PB. Emergence of multi-drugresistant Klebsiella pneumoniae in Neonatal Intensive Care Units: concern about antimicrobial policies. Research Journal of Recent Sciences 2012;1:275-280.

5. Sheth KV, Patel TK, Tripathi CB. Antibiotic sensitivity pattern in neonatal intensive care unit of a tertiary care hospital of India. Asian Journal of Pharmaceutical and Clinical Research 2012;5(3):46-50.

6. Meyer E, Schwab F, Jonas D. Surveillance of antimicrobial use and antimicrobial resistance in intensive care units (SARI): 1. antimicrobial use in german intensive care units. Intensive Care Med. 2004;30(6):1089-96.

7. Fridkin SK, Steward CD, Edwards JR. Surveillance of antimicrobial use and antimicrobial resistance in United
States hospitals: Project ICARE phase 2. Project intensive care antimicrobial resistance epidemiology (ICARE) hospitals. Clin Infect Dis. 1999;29(2):245-52.

8. Dworzack DL, Pugsley MP, Sanders CC. Emergence of resistance in gram negative bacteria during therapy with expanded-spectrum cephalosporins. Eur J Clin Microbiol. 1987;6(4):456-9.

9. Webb $\mathrm{CH}$. Antibiotic resistance associated with selective decontamination of the digestive tract. J Hosp Infect. 1992;22(1):1-5.

10. Yves Liem TB, Krediet TG, Andre' Fleer, Egberts TCG, Rademaker CMA. Variation in antibiotic use in neonatal intensive care units in the Netherlands. $J$ Antimicrob Chemother 2010;65(6):1270-5.

11. Badar VA, Navale SB. Study of Prescribing Pattern of Antimicrobial Agents in Medicine Intensive Care Unit of a Teaching Hospital in Central India. JAPI 2012; 60:20-23.

12. Tschudy MM, Arcara KM. The Johns Hopkins Hospital, The Harriet Lane Handbook, 19 ${ }^{\text {th }}$ Ed.2012.

13. Promoting rational use of medicines: core components, WHO Policy Perspectives on Medicines, September 2002 World Health Organization Geneva.

14. Ambwani S, Mathur AK. Rational drug use. Health Administrator. Chapter 2;19(1):5-7.

15. Cloherty J, Etic C, Anne R, Stark AR. A manual of neonatal care. $7^{\text {th }}$ Ed. Wolters Kluwer Health. Lippincott Willians and Wilkins.

16. Peter O, Pharoah D. Outcome Prediction in Greek Neonatal Intensive Care Units. J of American Academy of Paediatrics 1998;101(6):1070

17. Dorling JS, Field DJ, Manktelow B. Neonatal disease severity scoring systems. Arch Dis Child Fetal Neonatal Ed 2005;90:F11-F16.

\section{How to cite this article?}

Borade S, Ghodki S, Bhore A, Tiwari S, Jahagirdar S, Bansod K. Evaluation of antimicrobial prescription pattern in Neonatal Intensive care unit of tertiary care teaching hospital. Int J Med Res Rev 2014;2(5):474-479. doi:10.17511/ijmrr.2014.i05.12 